Bull. Austral. Math. Soc.

VOL. $62(2000) \quad[211-220]$

\title{
BEST CONSTANTS FOR TENSOR PRODUCTS OF BERNSTEIN, SZÁSZ AND BASKAKOV OPERATORS
}

\author{
Jesús de la Cal and Ana M. Valle
}

\begin{abstract}
We consider tensor product operators and discuss their best constants in preservation inequalities concerning the usual moduli of continuity. In a previous paper, we obtained lower and upper bounds on such constants, under fairly general assumptions on the operators. Here, we concentrate on the $l_{\infty}$-modulus of continuity and three celebrated families of operators. For the tensor product of $k$ identical copies of the Bernstein operator $B_{n}$, we show that the best uniform constant coincides with the dimension $k$, when $k \geqslant 3$, while, in case $k=2$, it lies in the interval $[2,5 / 2]$ but depends upon $n$. Similar results also hold when $B_{n}$ is replaced by a univariate Szász or Baskakov operator. The three proofs follow the same pattern, a crucial ingredient being some special properties of the probability distributions involved in the mentioned operators, namely: the binomial, Poisson, and negative binomial distributions.
\end{abstract}

\section{INTRODUCTION AND MAIN RESULTS}

The preservation of global smoothness by positive linear operators and the best constants involved have been actively investigated by several authors during the last few years. We refer, for instance, to $[\mathbf{1}, \mathbf{2}, \mathbf{3}, \mathbf{4}, \mathbf{5}, \mathbf{6}, \mathbf{7}, \mathbf{8}, \mathbf{9}]$ and the references therein, where different approaches can be found. In particular, when dealing with operators of probabilistic type (also called Bernstein type operators), the approach in $[\mathbf{1}, \mathbf{2}, \mathbf{6}, \mathbf{7}, \mathbf{9}]$ based on representations of the operators in terms of suitable stochastic processes has shown to be adequate and fruitful.

Following this spirit, we have given in [7] a systematic account of general results for tensor products of Bernstein type operators including explicit formulae for the best constants in terms of the underlying stochastic processes, as well as numerical lower and upper bounds when the operators fulfill appropriate conditions. Among other things, we showed the following:

Let $L$ be a Bernstein-type operator over the interval $I$ (either $[0,1]$ or $[0, \infty)$ ) allowing for a representation of the form

$$
L f(x):=E f(Z(x)), \quad x \in I, f \in \mathcal{L},
$$

Received 1st November, 1999

Research supported by the University of the Basque Country, and by the DGESIC PB98-1577-C02-02 Grant.

Copyright Clearance Centre, Inc. Serial-fee code: 0004-9727/00 \$A2.00+0.00. 
where (here and hereafter) $E$ denotes mathematical expectation, $Z:=\{Z(x): x \in$ $I\}$ is an integrable $I$-valued stochastic process, starting at 0 and having stationary increments, and $\mathcal{L}$ stands for the domain of $L$, that is, the set of all real valued functions on $I$ for which the right-hand side in (1) makes sense. Denote by $L^{\langle k\rangle}:=L \otimes \cdots \otimes L$ the tensor product of $k$ copies of $L$, that is, the $k$-dimensional operator given by

$$
L^{\langle k\rangle} f(\mathbf{x}):=E f\left(Z_{1}\left(x_{1}\right), \ldots, Z_{k}\left(x_{k}\right)\right), \quad \mathbf{x}:=\left(x_{1}, \ldots, x_{k}\right) \in I^{k}, f \in \mathcal{L}^{\langle k\rangle},
$$

where $Z_{i}:=\left\{Z_{i}(x): x \in I\right\}(i=1, \ldots, k)$ are $k$ stochastically independent copies of $Z$ defined on the same probability space. If $C^{(k)}(\delta)$ is the best positive constant (not depending upon $f$ ) such that

$$
\omega\left(L^{\langle k\rangle} f ; \delta\right) \leqslant C^{\langle k\rangle}(\delta) \omega(f ; \delta), \quad f \in \mathcal{L}^{\langle k\rangle}, \delta \in I-\{0\},
$$

where $\omega(f ; \cdot)$ stands for the usual modulus of continuity of $f$ with respect to the $l_{\infty}$ norm on $\mathbb{R}^{k}$, then

$$
C^{\langle k\rangle}(\delta)=E\left\lceil\frac{\max \left(Z_{1}(\delta), \ldots, Z_{k}(\delta)\right)}{\delta}\right\rceil,
$$

where

$$
\lceil a\rceil:=\text { the smallest integer not less than } a .
$$

Moreover, under the additional assumptions:

(a) $E Z(x)=x(x \in I)$, and

(b) $\lim _{x \downarrow 0} P(Z(x)=0)=1$,

we have

$$
k=\lim _{\delta \downarrow 0} C^{\langle k\rangle}(\delta) \leqslant \sup _{\delta \in I-\{0\}} C^{\langle k\rangle}(\delta) \leqslant k+1 .
$$

The preceding results apply to many operators usually considered in approximation theory. However, the theoretical computation of the best uniform constant $\sup C^{\langle k\rangle}(\delta)$, or the sharpening of (3), requires specific techniques adapted to the $\delta \in I-\{0\}$

particular case under consideration.

In the present paper, we focus our attention on three of the most celebrated families of operators (Bernstein, Szász, and Baskakov operators), and establish some striking facts relating the dimension with the corresponding best uniform constants.

The next section contains the statements of the main results. The proofs are given in Section 4, and they are based on two main ingredients: on the one hand, the general 
relations (2) and (3) above; on the other hand, some special properties of the probability distributions involved in the mentioned operators (namely: the binomial, Poisson, and negative binomial distributions) leading to the auxiliary results collected in Section 3 . Since the three proofs follow the same pattern, we only give complete details in the case of the Bernstein operator. Finally, some concluding remarks close the paper.

\section{MAIN RESUlts}

BERNSTEIN OPERATORS. The $n$th Bernstein operator $B_{n}$ over the interval $[0,1]$ is given by

$$
B_{n} f(x):=\sum_{j=0}^{n} f(j / n) p_{n, j}(x)=E f\left(\frac{1}{n} \sum_{i=1}^{n} 1_{\left(U_{i} \leqslant x\right)}\right),
$$

where the $p_{n, j}(x)$ are the weights of the binomial distribution with parameters $n, x$, that is,

$$
p_{n, j}(x):=\left(\begin{array}{c}
n \\
j
\end{array}\right) x^{j}(1-x)^{n-j}
$$

and $U_{1}, U_{2}, \ldots$, are independent and uniformly distributed random variables on the interval $[0,1]$. Since $B_{n}$ depends on the parameter $n$, we shall denote by $C_{n}^{\langle k\rangle}(\delta)$ the best constant for the tensor product $B_{n}^{\langle k\rangle}$ of $k$ copies of $B_{n}$ (the same convention will be used in the other cases considered below). It is known $[2,3]$ that, for $k=1$ and all $n \geqslant 1$,

$$
\sup _{0<\delta \leqslant 1} C_{n}^{\langle 1\rangle}(\delta)=2
$$

In this paper, we show the following.

THEOREM 1. In the case of Bernstein operators, we have for all $n \geqslant 1$ :

(a) If $k \geqslant 3$, then $\sup _{0<\delta \leqslant 1} C_{n}^{\langle k\rangle}(\delta)=\lim _{\delta \downarrow 0} C_{n}^{\langle k\rangle}(\delta)=k$.

(b) If $k=2$, then $2=\lim _{\delta \downarrow 0} C_{n}^{\langle 2\rangle}(\delta) \leqslant \sup _{0<\delta \leqslant 1} C_{n}^{\langle 2\rangle}(\delta) \leqslant 5 / 2$.

REMARK 1. Unlike the cases $k=1$ and $k \geqslant 3, \sup _{0<\delta \leqslant 1} C_{n}^{(2)}(\delta)$ does depend upon $n$ (see Section 5 below). It should also be noticed that, for tensor products of different Bernstein operators, the statement analogous to Theorem 1(a) fails to be true (see [7]). SzÁsz-Mirakyan operators. For $t>0$, the Szász operator $S_{t}$ over $[0, \infty)$ is defined by

$$
S_{t} f(x):=\sum_{k=0}^{\infty} f(k / t) \pi_{t, j}(x)=E f\left(\frac{N(t x)}{t}\right)
$$


where the $\pi_{t, j}(x)$ are the weights of the Poisson distribution with parameter $t x$

$$
\pi_{t, j}(x):=e^{-t x} \frac{(t x)^{j}}{j !}
$$

and $\{N(u): u \geqslant 0\}$ is a standard Poisson process. In [9], it is shown that, for all $t>0$,

$$
\sup _{\delta>0} C_{t}^{\langle 1\rangle}(\delta)=2-e^{-1}
$$

In the present paper, we prove the following.

THEOREM 2. In the case of Szász operators, we have for all $t>0$ :

(a) If $k \geqslant 3$, then $\sup _{\delta>0} C_{t}^{\langle k\rangle}(\delta)=\lim _{\delta \downarrow 0} C_{t}^{\langle k\rangle}(\delta)=k$.

(b) If $k=2$, then $2=\lim _{\delta \downarrow 0} C_{t}^{\langle 2\rangle}(\delta) \leqslant \sup _{\delta>0} C_{t}^{\langle 2\rangle}(\delta) \leqslant 5 / 2$.

REMARK 2. The best uniform constant $\sup _{\delta>0} C_{t}^{\langle k\rangle}(\delta)$ is independent of $t>0$ (for each $k \geqslant 1$ ), as readily follows from the application of $(2)$ to the situation at hand. In Section 5 , we state a conjecture about the exact value of such a constant, in the case $k=2$.

BASKAKov OPERATORs. For $t>0$, the Baskakov operator $H_{t}$ over $[0, \infty)$ is defined by

$$
H_{t} f(x):=\sum_{k=0}^{\infty} f(k / t) b_{t, j}(x)=E\left(\frac{N\left(x U_{t}\right)}{t}\right),
$$

where the $b_{t, j}(x)$ are the weights of the negative binomial distribution with parameters $t, x$, that is,

$$
b_{t, j}(x):=\left(\begin{array}{c}
t+j-1 \\
j
\end{array}\right) \frac{x^{j}}{(1+x)^{t+j}},
$$

$\{N(u): u \geqslant 0\}$ is a standard Poisson process, and $U_{t}$ is a random variable having the gamma distribution with parameters $t, 1$, and independent of the Poisson process. It was shown in [2] that

$$
\sup _{t>0} \sup _{\delta>0} C_{t}^{\langle 1\rangle}(\delta)=2 \text {. }
$$

Our last main result in the present paper is stated as follows.

THEOREM 3. In the case of Baskakov operators, we have for all $t>0$ and $k \geqslant 2$

$$
\sup _{\delta>0} C_{t}^{\langle k\rangle}(\delta) \leqslant k+1-\alpha_{t, k}
$$

where

$$
\alpha_{t, k}:=\min \left\{1, \frac{\left(\begin{array}{c}
k \\
2
\end{array}\right) t}{t k+1}\right\} .
$$

Combining (3) with the preceding theorem, we obtain the following. 
COROLLARY. In the setting of Theorem 3, we have:
(a) $2=\lim _{\delta \downarrow 0} C_{t}^{\langle 2\rangle}(\delta) \leqslant \sup _{\delta>0} C_{t}^{(2\rangle}(\delta) \leqslant 3-(t /(2 t+1))$.
(b) $3=\lim _{\delta \downarrow 0} C_{t}^{\langle 3\rangle}(\delta) \leqslant \sup _{\delta>0} C_{t}^{(3)}(\delta) \leqslant 4-(3 t /(3 t+1))$.
(c) For $k \geqslant 4$ and $t \geqslant 2 / k(k-3), \sup _{\delta>0} C_{t}^{\langle k\rangle}(\delta)=\lim _{\delta \downarrow 0} C_{t}^{\langle k\rangle}(\delta)=k$.

\section{AuXiliary RESUlts}

In this section, we collect the auxiliary results to be used in the proofs of Theorems 1-3. They all are concerned with the expected maximum of a finite set of independent identically distributed random variables.

We shall use the following notation: $Y_{1}(x), \ldots, Y_{k}(x)(k \geqslant 2)$ are independent random variables taking values in the set of nonnegative integers, and having the same probability distribution depending on the parameter $x \in I$ (= either the interval $(0,1]$ or the interval $(0, \infty))$. We also set

$$
\begin{aligned}
Y(x) & :=\max \left\{Y_{i}(x): i=1, \ldots, k\right\}, \\
Y_{i}^{*}(x) & :=\max \left\{Y_{j}(x): j \neq i\right\}, \quad i=1, \ldots, k, \\
Y_{i, j}^{*}(x) & :=\left\{\begin{array}{ll}
\max \left\{Y_{l}(x): l \neq i, j\right\} & \text { if } k \geqslant 3 \\
0 & \text { if } k=2,
\end{array} \quad 1 \leqslant i<j \leqslant k .\right.
\end{aligned}
$$

Our first lemma is an easy exercise in probability theory. However, for the sake of completeness, we include the details of the proof.

LEMMA 1. We have, for all $x \in I$,

$$
E Y(x)=\sum_{l=0}^{\infty}\left[1-P^{k}\left(Y_{1}(x) \leqslant l\right)\right]
$$

Proof: We have,

$$
\begin{aligned}
E Y(x) & =\sum_{l=1}^{\infty} l P(Y(x)=l)=\sum_{l=1}^{\infty} P(Y(x) \geqslant l) \\
& =\sum_{l=0}^{\infty}(1-P(Y(x) \leqslant l))=\sum_{l=0}^{\infty}\left[1-P^{k}\left(Y_{1}(x) \leqslant l\right)\right] .
\end{aligned}
$$

In Lemmas 2-4 below, the common distribution of the random variables $Y_{i}(x)$ is completely specified. 
LEMMA 2. Let $n \geqslant 1$ and $k \geqslant 2$ be fixed integers, and assume that the common distribution of the random variables $Y_{i}(x)$ is the binomial distribution with parameters $n$ and $x \in(0,1]$, that is,

$$
P\left(Y_{i}(x)=j\right)=p_{n, j}(x), \quad j=0, \ldots, n,
$$

where $p_{n, j}(x)$ is given in (4). If $\alpha_{k}$ is either $1 / 2$ or 1 , according to $k=2$ or $k \geqslant 3$, then the function

$$
h(x):=1-\alpha_{k}(1-x)^{n k}+\frac{E Y(x)}{n x}, \quad x \in(0,1],
$$

is nonincreasing, and

$$
\sup _{0<x \leqslant 1} h(x)=\lim _{x \downarrow 0} h(x)=k+1-\alpha_{k} .
$$

Proof: The nonincreasing character of $h(\cdot)$ will be established by showing that this function has a nonpositive derivative. For $x \in(0,1]$, it is clear that

$$
n x^{2} \frac{d}{d x} h(x)=\alpha_{k} k n^{2} x^{2}(1-x)^{n k-1}+x \frac{d}{d x} E Y(x)-E Y(x) .
$$

Since $\alpha_{k} k \leqslant\left(\begin{array}{l}k \\ 2\end{array}\right)$, we have

$$
\begin{aligned}
\alpha_{k} k n^{2} x^{2}(1-x)^{n k-1} & \leqslant \alpha_{k} k n^{2} x^{2}(1-x)^{n k-2} \\
& \leqslant\left(\begin{array}{c}
k \\
2
\end{array}\right)\left[p_{n, 1}(x)\right]^{2}\left[p_{n, 0}(x)\right]^{k-2} \\
& =\sum_{1 \leqslant i<j \leqslant k} P\left(Y_{i}(x)=1\right) P\left(Y_{j}(x)=1\right) P\left(Y_{i, j}^{*}(x)=0\right) .
\end{aligned}
$$

On the other hand, by Lemma 1 and the fact that

$$
x \frac{d}{d x} p_{n, j}(x)=j p_{n, j}(x)-(j+1) p_{n, j+1}(x), \quad j=0,1,2, \ldots
$$

we can write

$$
\begin{aligned}
x \frac{d}{d x} E Y(x) & =-\sum_{l=0}^{n-1} k P^{k-1}\left(Y_{1}(x) \leqslant l\right) x \frac{d}{d x} P\left(Y_{1}(x) \leqslant l\right) \\
& =-\sum_{l=0}^{n-1} k P^{k-1}\left(Y_{1}(x) \leqslant l\right)\left\{\sum_{j=0}^{l}\left[j p_{n, j}(x)-(j+1) p_{n, j+1}(x)\right]\right\} \\
& =\sum_{l=0}^{n-1} k P^{k-1}\left(Y_{1}(x) \leqslant l\right)(l+1) P\left(Y_{1}(x)=l+1\right) \\
& =\sum_{l=0}^{n-1}(l+1)\left\{\sum_{i=1}^{k} P\left(Y_{i}^{*}(x) \leqslant l\right) P\left(Y_{i}(x)=l+1\right)\right\}
\end{aligned}
$$


For each $l=0,1, \ldots$, the $k+\left(\begin{array}{l}k \\ 2\end{array}\right)$ events

$$
\begin{aligned}
\left\{Y_{i}(x)=l+1\right\} \cap\left\{Y_{i}^{*}(x) \leqslant l\right\}, & 1 \leqslant i \leqslant k, \\
\left\{Y_{i}(x)=l+1\right\} \cap\left\{Y_{j}(x)=l+1\right\} \cap\left\{Y_{i, j}^{*}(x) \leqslant l\right\}, & 1 \leqslant i<j \leqslant k
\end{aligned}
$$

are pairwise disjoint, and they all are included in the event $\{Y(x)=l+1\}$. Therefore, we obtain from (9) and (11)

$$
\begin{aligned}
\alpha_{k} k n^{2} x^{2}(1-x)^{n k-1}+x \frac{d}{d x} E Y(x) & \leqslant \sum_{l=0}^{n-1}(l+1) P(Y(x)=l+1) \\
& =E Y(x),
\end{aligned}
$$

which, by (8), implies $\frac{d}{d x} h(x) \leqslant 0$.

Finally, from l'Hôpital's rule and the above calculations, we have

$$
\begin{aligned}
\lim _{x \downarrow 0} \frac{E Y(x)}{n x} & =\lim _{x \downarrow 0} k \sum_{l=0}^{n-1} P^{k-1}\left(Y_{1}(x) \leqslant l\right)\left(\begin{array}{c}
n-1 \\
l
\end{array}\right) x^{l}(1-x)^{n-l-1} \\
& =k
\end{aligned}
$$

showing (7), and completing the proof of Lemma 2.

It is immediately checked that (10) remains true when the functions $p_{n, j}(\cdot)$ are replaced by the functions $\pi_{t, j}(\cdot)$ given in (5) (respectively, the functions $b_{t, j}(\cdot)$ given in (6)), for all $t, x>0$. Using this fact, the proof of Lemma 3 (respectively, Lemma 4) follows exactly the same pattern as the proof of Lemma 2, and we therefore omit the details.

LEMMA 3. Let $t>0, k \geqslant 2$, and assume that the common distribution of the random variables $Y_{i}(x)$ is the Poisson distribution with parameter $t x(x \in(0, \infty))$, that is,

$$
P\left(Y_{i}(x)=j\right)=\pi_{t, j}(x), \quad j=0,1, \ldots,
$$

where $\pi_{t, j}(x)$ is given in (5). If $\alpha_{k}$ is the same as in Lemma 2, then the function

$$
h(x):=1-\alpha_{k} e^{-t k x}+\frac{E Y(x)}{t x}, \quad x \in(0, \infty)
$$

is nonincreasing, and

$$
\sup _{x>0} h(x)=\lim _{x \downarrow 0} h(x)=k+1-\alpha_{k}
$$


LEMmA 4. Let $t>0, k \geqslant 2$, and assume that the common distribution of the random variables $Y_{i}(x)$ is the negative binomal distribution with parameters $t$ and $x \in(0, \infty)$, that is,

$$
P\left(Y_{i}(x)=j\right)=b_{t, j}(x), \quad j=0,1, \ldots,
$$

where $b_{t, j}(x)$ is given in (6). If $\alpha_{t, k}$ is the same as in the statement of Theorem 3 , then the function

$$
h(x):=1-\alpha_{t, k}(1+x)^{-(t k+1)}+\frac{E Y(x)}{t x}, \quad x \in(0, \infty),
$$

is nonincreasing, and

$$
\sup _{x>0} h(x)=\lim _{x \downarrow 0} h(x)=k+1-\alpha_{t, k}
$$

\section{Proofs of Theorems $1-3$}

4.1 Proof of Theorem 1. Let $n \geqslant 1$ and $k \geqslant 2$. Since (3) holds true when $I$ and $C^{\langle k\rangle}(\delta)$ are replaced by $[0,1]$ and $C_{n}^{\langle k\rangle}(\delta)$, respectively, we only need to show that

$$
\sup _{\delta \in(0,1]} C_{n}^{\langle k\rangle}(\delta) \leqslant k+1-\alpha_{k},
$$

where $\alpha_{k}$ is the same as in Lemma 2 .

The application of formula (2) to the situation at hand yields

$$
C_{n}^{\langle k\rangle}(\delta)=E\left\lceil\frac{Y(\delta)}{n \delta}\right\rceil, \quad \delta \in(0,1]
$$

where $Y(\cdot)$ is the same as in Lemma 2.

Using the inequality $E\lceil U\rceil \leqslant P(U>0)+E U$, (which holds for every nonnegative random variable $U)$, and the fact that $\alpha_{k} \leqslant 1$, we therefore have, for every $\delta \in(0,1]$,

$$
\begin{aligned}
C_{n}^{\langle k\rangle}(\delta) & \leqslant 1-P(Y(\delta)=0)+\frac{E Y(\delta)}{n \delta} \\
& \leqslant 1-\alpha_{k}(1-\delta)^{n k}+\frac{E Y(\delta)}{n \delta} \\
& =h(\delta),
\end{aligned}
$$

where $h(\cdot)$ is defined as in the statement of Lemma 2. The conclusion follows from this lemma.

4.2 Proofs OF TheOREMS 2 AND 3: In the setting of Theorem 2 (respectively, Theorem 3), formula (2) gives

$$
C_{t}^{\langle k\rangle}(\delta)=E\left\lceil\frac{Y(\delta)}{t \delta}\right\rceil, \quad t, \delta>0
$$

where $Y(\cdot)$ is the same as in Lemma 3 (respectively, Lemma 4). The same argument as above yields the corresponding conclusions. 


\section{CONCLUDING REMARKS}

5.1 BERNSTEIN OPERATORS. In the two-dimensional case, we have

$$
C_{n}^{\langle 2\rangle}(\delta)=\sum_{j=1}^{n}\left\lceil\frac{j}{n \delta}\right\rceil\left\{\left(\sum_{i=0}^{j} p_{n, i}(\delta)\right)^{2}-\left(\sum_{i=0}^{j-1} p_{n, i}(\delta)\right)^{2}\right\}, \quad 0<\delta \leqslant 1 .
$$

Therefore,

$$
\sup _{0<\delta \leqslant 1} C_{1}^{\langle 2\rangle}(\delta) \leqslant \sup _{0<\delta \leqslant 1}\left(1+\frac{1}{\delta}\right)\left(1-(1-\delta)^{2}\right)=\frac{9}{4}
$$

and

$$
\sup _{0<\delta \leqslant 1} C_{2}^{\langle 2\rangle}(\delta) \geqslant \lim _{\delta \uparrow 1 / 2} C_{2}^{\langle 2\rangle}(\delta)=\frac{37}{16}
$$

(It can be checked that the preceding inequalities are actually equalities.) Thus, the best uniform constant $C_{n}^{\langle 2\rangle}:=\sup _{0<\delta \leqslant 1} C_{n}^{\langle 2\rangle}(\delta)$ depends upon $n$, as it was noticed in Remark 1.

It should also be remarked that, by managing on a computer the functions $C_{n}^{\langle 2\rangle}(\cdot)$, one can get empirical evidence supporting the following two conjectures:

ConJECTURE 1 . The sequence $\left\{C_{n}^{\langle 2\rangle}: n \geqslant 1\right\}$ is nondecreasing.

CONJECTURE 2. For each $n \geqslant 2, C_{n}^{\langle 2\rangle}$ equals

$$
\lim _{\delta \uparrow 1 / n} C_{n}^{\langle 2\rangle}(\delta)=1-\left(1-\frac{1}{n}\right)^{2 n}+\sum_{j=0}^{n-1}\left[1-\left(\sum_{i=0}^{j}\left(\begin{array}{c}
n \\
i
\end{array}\right) \frac{(n-1)^{n-i}}{n^{n}}\right)^{2}\right] .
$$

However, we have been unable so far to find a theoretical proof for such assertions. Thus, they will remain here as open problems. Observe that, if both conjectures are true, then

$$
\sup _{n \geqslant 1} C_{n}^{\langle 2\rangle}=1-e^{-2}+\sum_{j=0}^{\infty}\left[1-e^{-2}\left(\sum_{i=0}^{j} \frac{1}{i !}\right)^{2}\right]=2.3884 \ldots
$$

5.2 SzÁsz Operators. Let $Y(\delta)$ be the random variable in Lemma 3 corresponding to $t=1$ and $k=2$. Then, for the two-dimensional Szász operator $S_{1} \otimes S_{1}$, we have

$$
\begin{aligned}
\lim _{\delta \uparrow 1} C_{1}^{\langle 2\rangle}(\delta) & =\lim _{\delta \uparrow 1} E\left[\frac{Y(\delta)}{\delta}\right]=\sum_{l=1}^{\infty}(l+1) P(Y(1)=l) \\
& =1-e^{-2}+\sum_{l=0}^{\infty}\left[1-e^{-2}\left(\sum_{j=0}^{l} \frac{1}{j !}\right)^{2}\right] .
\end{aligned}
$$

Observe that this value coincides with the right-hand side in (12). Recalling Remark 2, we state the following. 
CONJECTURE 3. In the case of Szász operators, we have for all $t>0$

$$
\sup _{\delta>0} C_{t}^{(2)}(\delta)=\sup _{\delta>0} C_{1}^{\langle 2\rangle}(\delta)=\lim _{\delta \uparrow 1} C_{1}^{(2)}(\delta)
$$

\section{REFERENCES}

[1] J.A. Adell and J. de la Cal, 'Using stochastic processes for studying Bernstein-type operators', Rend. Circ. Mat. Palermo (2) Suppl. 33 (1993), 125-141.

[2] J.A. Adell and A. Pérez-Palomares, 'Best constants in preservation inequalities concerning the first modulus and Lipschitz classes for Bernstein-type operators', J. Approx. Theory 93 (1998), 128-139.

[3] G.A. Anastassiou, C. Cottin and H.H. Gonska, 'Global smoothness of approximating functions', Analysis 11 (1991), 43-57.

[4] G.A. Anastassiou, C. Cottin and H.H. Gonska, 'Global smoothness preservation by multivariate approximation operators', in Israel Mathematical Conference Proceedings, Vol. $I V$, (S. Baron and D. Leviatan, Editors) (Bar-Ilan University, Ramat-Gan, 1991), pp. 31-44.

[5] C. Cottin and H.H. Gonska, 'Simultaneous approximation and global smoothness preservation', Rend. Circ. Mat. Palermo (2) Suppl. 33 (1993), 259-279.

[6] J. de la Cal and A.M. Valle, 'Best constants in global smoothness preservation inequalities for some multivariate operators', J. Approx. Theory 97 (1999), 158-180.

[7] J. de la Cal and A.M. Valle, 'Global smoothness preservation by multivariate Bernstein-type operators', in Handbook on Analytic Computational Methods in Applied Mathematics (CRC Press LLC, Boca Raton) (to appear).

[8] W. Kratz and U. Stadtmüller, 'On the uniform modulus of continuity of certain discrete approximation operators', J. Approx. Theory 54 (1988), 326-337.

[9] A. Pérez-Palomares, 'Global smoothness preservation properties for generalized SzászKantorovich operators', (preprint).

Departamento de Matemática Aplicada y Estadística

e Investigación Operativa

Facultad de Ciencias

Universidad del Pais Vasco

Apartado 644, 48080 Bilbao

Spain

e-mail: mepcaagj@lg.ehu.es

mepvamaa@lg.ehu.es 